\title{
CARACTERÍSTICAS UNIVERSITARIAS ASOCIADAS A LA ADJUDICACIÓN DE PLAZAS PARA OBSTETRAS EN EL SERVICIO RURAL PERUANO
}

\author{
UNIVERSITY CHARACTERISTICS ASSOCIATED WITH THE AWARD OF PLACES FOR MIDWIVES IN \\ THE PERUVIAN RURAL SERVICE \\ Elizabeth Llanos-Najarro1,a, Dulce Villafuerte-Cooban,a, Victor Moquillaza-Alcántaraª,b
}

\begin{abstract}
RESUMEN
Objetivo: Determinar las características universitarias asociadas a la adjudicación de plazas para obstetras en el Servicio Rural y Urbano Marginal en Salud (SERUMS), 2019. Métodos: Análisis de base secundaria obtenida del Ministerio de Salud peruano, donde se seleccionaron 506 licenciados de obstetricia que rindieron el examen nacional de obstetricia (ENAOBS) y que hayan postulado al SERUMS en la convocatoria 2019-2. Se evaluaron las características de las universidades de donde procedían los obstetras, el puntaje obtenido en el examen nacional y universitario y si fue apto para el SERUMS. Para el análisis bivariado se utilizaron las pruebas de T de Student, ANOVA y Correlación de Pearson, mientras que para ajustar el modelo se realizó una regresión logística, considerando un nivel de confianza del 95\%. Resultados: El egresado de obstetricia proviene en su mayoría de una universidad privada (66,01\%), de la región Lima $(41,7 \%)$ y postula a la modalidad remunerada $(99,41 \%)$. Se halló que el 49,61\% [IC95\%:45,23-53,98] fueron aptos para el SERUMS. La calificación universitaria promedio fue de $13,87 \pm 1,35$ y la del ENAOBS de $10,93 \pm 2,02$, las cuales mostraron una correlación significativa $(p<0,001)$ que tuvo una mayor pendiente en egresados de universidad pública $(R=0,56)$. El pertenecer a una universidad pública $(O R=2,62)$ y estudiar en Lima $(O R=1,49)$ se asociaron significativamente $(p<0,01)$ al estar apto para realizar el SERUMS en obstetricia. Conclusión: Ser egresado en obstetricia de una universidad pública y estudiar en Lima brinda mayor probabilidad de ser apto para adjudicar una plaza en el SERUMS.
\end{abstract}

Palabras clave: Evaluación educacional; Educación médica; Obstetricia; Perú (fuente: DeCS BIREME).

\begin{abstract}
Objective: To determine the university characteristics associated with the allocation of place for obstetricians in the Rural and Urban Marginal Service in Health (SERUMS), 2019. Methods: Secondary base analysis obtained from the Peruvian Ministry of Health, where 506 obstetrics licenses were selected who submitted the national obstetrics exam (ENAOBS) and who have applied to SERUMS in the 2019-2 call. The characteristics of the universities where the obstetricians come from, the score obtained in the national and university exam and if it is suitable for SERUMS were evaluated. For the bivariate analysis it will be used in the Student's T, ANOVA and Pearson's Correlation tests, while to adjust the model a logistic regression was performed, a 95\% confidence level. Results: The obstetrics graduate visited mostly from a private university (66.01\%), from the Lima region (41.7\%) and applied for the paid modality (99.41\%). It was found that $49.61 \%$ [95\% Cl: 45.23-53.98] were suitable for SERUMS. The average university qualification was 13.87 \pm 1.35 and that of the ENAOBS was $10.93 \pm 2.02$, which had a significant correlation $(p<0.001)$ that had a greater slope in graduates of public university $(R=0.56)$. Belonging to a public university $(O R=2.62)$ and studying in Lima $(O R=1.49)$ were significantly associated $(p<0.01)$ to be able to perform SERUMS in obstetrics. Conclusion: Being a graduate in obstetrics from a public university and studying in Lima gives you a better chance of being able to award a place in the SERUMS.
\end{abstract}

Key words: Educational Measurement; Health education; Midwifery; Peru (source: MeSH NLM).

${ }^{1}$ Centro Federado de Obstetricia, Universidad Nacional Mayor de San Marcos, Lima-Perú.

2 Facultad de Salud Pública y Administración, Universidad Peruana Cayetano Heredia, Lima-Perú.

a Estudiante de obstetricia.

b Licenciado en obstetricia, Becario de la Maestría en Informática Biomédica en Salud Global.

Citar como: Elizabeth Llanos-Najarro, Dulce Villafuerte-Cooban, Victor Moquillaza-Alcántara. Características universitarias asociadas a la adjudicación de plazas para obstetras en el servicio rural peruano. Rev. Fac. Med. Hum. Abril 2020; 20(2):240-245. DOI 10.25176/RFMH.v20i2.2897 


\section{INTRODUCCIÓN}

La universidad es aquella institución que, entre sus funciones, busca formar académicamente a futuros profesionales, brindando así recurso humano competente en determinadas áreas ${ }^{(1,2)}$. El sistema universitario peruano está conformado por 51 universidades públicas y 92 privadas, las cuales vienen siendo supervisadas por la Superintendencia Nacional de Educación (SUNEDU), a fin de vigilar y garantizar la calidad universitaria que se viene impartiendo ${ }^{(3)}$.

El año 2016 el Ministerio de Salud oficializó el examen nacional de obstetricia (ENAOBS), el cual es un instrumento que permite evaluar el conocimiento de los profesionales obstetras. Además, los resultados determinan quienes podrían adjudicar plazas en el Servicio Rural y Urbano Marginal de Salud (SERUMS). De esta manera se busca promover y garantizar una debida formación del profesional obstetra y, por consecuencia, garantizar una mejor atención en salud sexual y reproductiva ${ }^{(4,5)}$.

Por otro lado, el SERUMS es la atención que debe brindar el profesional de la salud por un año en una población vulnerable, para poder ocupar un cargo en instituciones públicas. Las investigaciones al respecto se han centrado en dos tópicos claros: i) los problemas y/o accidentes que ocurren en este proceso(6-8) y ii) el debate sobre si es necesaria su realización ${ }^{(9-11)}$. Sin embargo, a pesar de que actualmente llega a ser un requisito obligatorio, se desconocen cuáles son los elementos que favorecerían el ser apto para adjudicar una plaza; información que al aspirante a obstetra le serviría para cotejar qué características presenta la institución académica donde se formaría. Por lo cual, la investigación tuvo como objetivo determinar las características universitarias que se asocian al ser apto para adjudicar una plaza en el SERUMS.

\section{MÉTODOS}

\section{Diseño}

Estudio analítico transversal donde se utilizó una base secundaria de acceso libre del Ministerio de Salud peruano (MINSA) que reporta los resultados del concurso público para la adjudicación de plazas al Servicio Rural y Urbano Marginal en Salud (SERUMS) durante el $2019^{(12)}$.

\section{Población y muestra}

Se seleccionaron a los 506 profesionales de obstetricia que participaron en la convocatoria, los cuales provenían de las diversas regiones del Perú.

\section{Procedimientos y variables}

Se consideró como variable principal al ser apto o no para adjudicar una plaza en el SERUMS, el cual es generado en base a la calificación obtenida en el examen nacional de obstetricia y el promedio universitario que haya presentado, ambas bajo un sistema de puntaje vigesimal. Se considera, según el MINSA, como apto al profesional que tenga una calificación promedio aprobatorio mayor o igual a 11. Por otro lado, también se evaluó si el participante provenía de una universidad pública o privada, si se había cursado sus estudios en Lima, la región geográfica de procedencia (costa, sierra o selva), nacionalidad y la modalidad en la que postula (remunerado o equivalente).

La base de datos fue descargada en un formato de Excel, donde se evaluó la calidad de los datos obtenidos. Se eliminaron todos aquellos participantes que presenten ausencia o inconsistencia de datos, para luego exportarlo al software STATA. El análisis descriptivo de las variables categóricas se reportó mediante frecuencias y porcentajes, mientras que las variables numéricas se reportaron mediante medias aritméticas y desviación estándar, debido a la distribución normal que presentaban, la cual fue evaluada mediante la prueba Shapiro-Wilk.

\section{Análisis estadística}

En el análisis inferencial, la variación de las medias fue evaluada mediante la prueba T de Student o ANOVA, de acuerdo a si existían 2 o más grupos de comparación. Asimismo, la correlación entre las calificaciones del ENAOBS y el promedio universitario fueron evaluados mediante la prueba de Correlación de Pearson. El análisis crudo de variables categóricas se realizó mediante la prueba Chi Cuadrado de Pearson y el análisis ajustado mediante una Regresión Logística, donde se reportaron OR (Odds Ratio), crudo y ajustado, y sus respectivos intervalos de confianza. Todo análisis consideró un 95\% de nivel de confianza.

\section{Consideraciones éticas}

El estudio no requirió la presencia de un consentimiento informado puesto que los datos son de libre acceso; asimismo, debido a su naturaleza de análisis de base secundaria no fue necesaria la aprobación de un comité de ética institucional.

\section{RESULTADOS}

El en estudio se incluyeron 506 profesionales de obstetricia de los cuales el 49,61\% [IC95\%:45,23-53,98] logró ser apto para adjudicar una plaza en el servicio rural y urbanomarginal durante la convocatoria del 2019, en donde la calificación promedio al terminar la

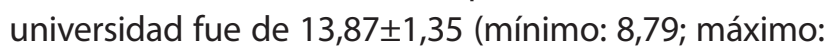
$17,56)$, mientras que la calificación promedio del examen nacional de obstetricia fue de 10,93 $\pm 2,02$ (mínimo: 4,02; máximo: 16,4). El participante proviene 
en su mayoría de una universidad privada (66,01\%), de la región Costa $(62,06 \%)$ y postula por modalidad remunerada $(99,41 \%)$. No se hallaron profesionales de nacionalidad extranjera. (Tabla 1)

En la Tabla 2, se observa que la calificación obtenida en el examen nacional de obstetricia es significativamente mayor cuando el participante proviene de una universidad pública $(p<0,001)$ y estudió en Lima $(p=0,005)$. Asimismo, el promedio obtenido en la universidad fue significativamente mayor cuando el participante había estudiado en Lima $(p<0,001)$ y provenía de la región Costa $(p=0,043)$. Por otro lado, la correlación entre la calificación universitaria presentó una mayor pendiente cuando el participante provenía de una universidad pública $(R=0,558)$ que cuando provenía de una privada $(R=0,425)$. (Figura 1)

Finalmente, el análisis ajustado mostró que el haber pertenecido a una universidad pública incrementa la probabilidad de ser apto para alcanzar una plaza en el Servicio Ruraly Urbano Marginal en obstetricia $(\mathrm{OR}=2,99$; $\mathrm{p}<0,001)$. Asimismo, otro factor predisponente al ser apto fue el haber estudiado en la capital del país $(\mathrm{OR}=2,26 ; \mathrm{p}=0,001)$ (Tabla 3).

Tabla 1. Características generales de los profesionales en obstetricia que postulan al Servicio Rural y Urbano Marginal en Salud (SERUMS), 2019.

N

\section{Tipo de universidad}

Pública

Privada

Sede en la capital

$\mathrm{Si}$

No

\section{Región geográfica}

Costa

Sierra

Selva

295

\section{Nacionalidad}

Peruano

Extranjero

\section{Modalidad}

Remunerado

Equivalente

3

\section{Apto para el SERUMS}

$\mathrm{Si}$

No

\section{Promedio universitario}

(Media \pm D.S)

\section{Calificación del examen nacional de obstetricia}

(Media \pm D.S)

$(10,93 \pm 2,02)$

Total 
Tabla 2. Calificaciones obtenidas en el examen nacional y en el promedio universitario según características institucionales, 2019.

\section{Calificación obtenida}

\section{Examen nacional de obstetricia}

\section{Promedio universitario*}

$\begin{array}{llllll}\text { Media } & \text { D.S } & \text { p valort } & \text { Media } & \text { D.S } & \text { p valort }\end{array}$

Tipo de universidad

Pública

Privada

11,74

10,51

11,23

10,72

10,89

11,02

10,49

Selva

\section{Universidad privada}

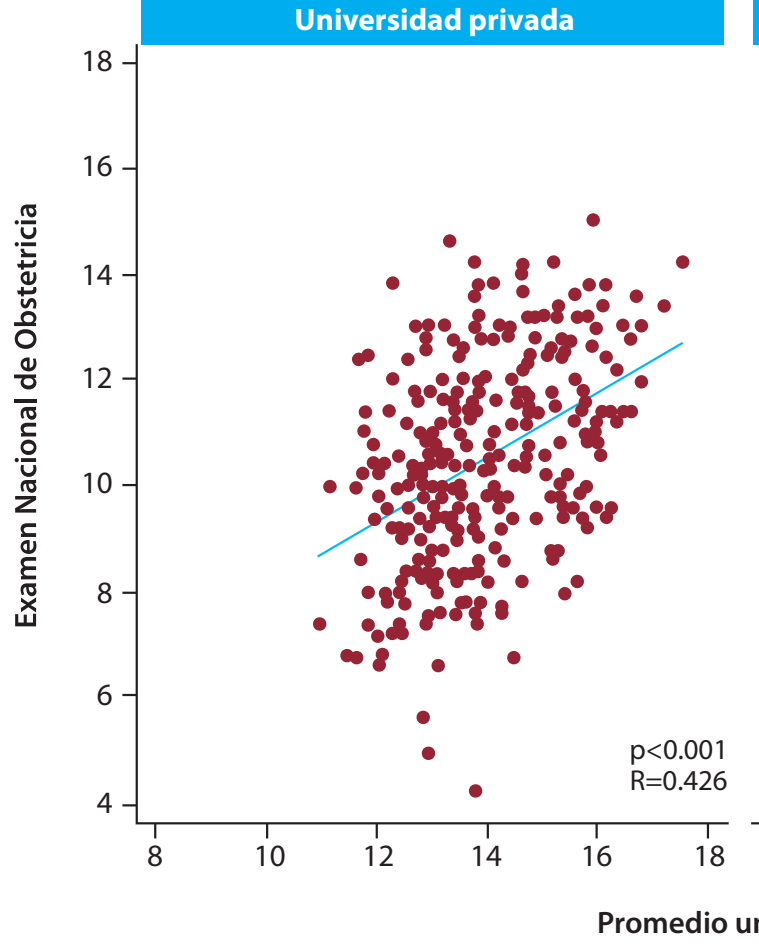

Evaluado mediante prueba de correlación de Pearson.

$13,72 \quad 1,42$

$13,94 \quad 1,31$

0,079

14,47

$13,43 \quad 1,20$

$<0,001$

0,005

$13,98 \quad 1,42$

$0,583+\dagger \quad 13,68 \quad 1,21$

1,11
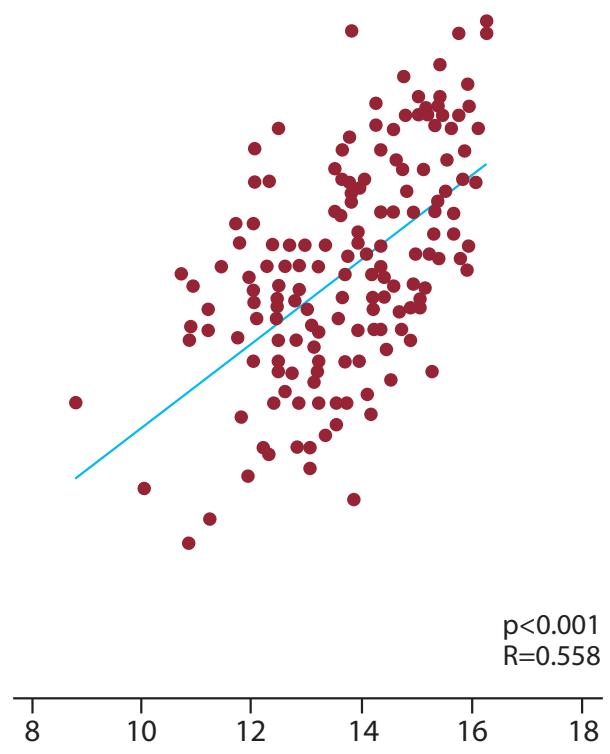

Figura 1. Correlación entre la calificación obtenida en el examen nacional de obstetricia y el promedio universitario de acuerdo con el tipo de universidad. 
Tabla 3. Características institucionales asociadas al estar apto para realizar el Servicio Rural y Urbano Marginal en Salud (SERUMS) en obstetricia.

\begin{tabular}{|c|c|c|c|c|c|c|}
\hline & \multicolumn{6}{|c|}{ Apto para realizar el SERUMS en obstetricia } \\
\hline & \multicolumn{3}{|c|}{ Análisis crudo † } & \multicolumn{3}{|c|}{ Análisis ajustado †† } \\
\hline & ORe & [IC95\%] & p valor & ORa & [IC95\%] & p valor \\
\hline \multicolumn{7}{|c|}{ Tipo de universidad } \\
\hline Pública & 2,62 & {$[1,75-3,91]$} & $<0,001$ & 2,99 & {$[1,99-4,49]$} & $<0,001$ \\
\hline Privada & \multicolumn{3}{|c|}{ Ref. } & \multicolumn{3}{|c|}{ Ref. } \\
\hline \multicolumn{7}{|c|}{ Sede en la capital } \\
\hline $\mathrm{Si}$ & 1,49 & {$[1,03-2,17]$} & 0,026 & 2,26 & {$[1,37-3,72]$} & 0,001 \\
\hline No & \multicolumn{3}{|c|}{ Ref. } & \multicolumn{3}{|c|}{ Ref. } \\
\hline \multicolumn{7}{|c|}{ Región geográfica } \\
\hline Costa & \multicolumn{3}{|c|}{ Ref. } & \multicolumn{3}{|c|}{ Ref. } \\
\hline Sierra & 1,09 & {$[0,75-1,61]$} & 0,621 & 1,45 & {$[0,86-2,44]$} & 0,160 \\
\hline Selva & 0,41 & {$[0,09-1,46]$} & 0,123 & 0,43 & {$[0,12-1,53]$} & 0,191 \\
\hline
\end{tabular}

ORc: Odds Ratio Crudo; ORa: Odds Ratio Ajustado; IC95\%: Intervalo de confianza al 95\%.

† Evaluado mediante la prueba Chi Cuadrado de Pearson.

†† Evaluado mediante la prueba de Regresión Logística.

$R 2=0,05 ; p<0,001$

\section{DISCUSIÓN}

Los resultados mostraron que el tener una formación en una universidad pública predispone a una mejor calificación en un examen nacional y un mayor promedio universitario. Esto difiere con algunos reportes que refieren que, debido al tipo de financiamiento, la universidad privada cuenta con mejor infraestructura, equipos y laboratorios ${ }^{(13)}$, lo cual favorecería el aprendizaje de sus estudiantes ${ }^{(14)}$; asimismo, se ha evidenciado que los estudiantes de universidad públicas presentan desventajas, como un mayor riesgo de desarrollar anemia ${ }^{(15)}$.

Sin embargo, un estudio realizado en Brasil menciona que los docentes de instituciones públicas muestran más experiencia en la enseñanza y asisten más frecuentementeaactividades deformación pedagógica; asimismo, en el sector educativo privado se suele tener mayor carga docente ${ }^{(16)}$. Con ello sugerimos que, en un ámbito general, la universidad privada brindaría mejores herramientas tecnológicas a sus estudiantes, mientras que la institución pública contaría con docentes mejor preparados, lo cual determinaría en mayor medida un mayor conocimiento en los estudiantes.

Por otro lado, el haber estudiado en la capital del país se mostró como un factor que predispone el tener mayor calificación y por ende estar apto para adjudicar una plaza. Esto puede deberse a que el Perú es una país con notoria desigualdad social que no incluye en su formación académica los conocimientos de pueblos andinos y amazónicos ${ }^{(17,18)}$, lo cual se traduce en presencia de mayores oportunidades educativas en la ciudad capital; esto debido a: i) la alta concentración de universidades, con lo cual existe una mayor variedad de opciones y costos educativos ${ }^{(19)}$ y ii) la considerable densidad de profesionales con estudios de posgrado que migran a la ciudad de Lima, la cual supera el $60 \%$ y refleja la distribución centralista e inequitativa del país ${ }^{(20)}$.

Finalmente, la región geográfica donde estudió el participante no llegó a ser un determinante en las calificaciones que obtuvo; al respecto no se ha hallado evidencia que compare en base a las mismas regiones (costa, sierray selva), perosi se ha realizado bajo entornos rurales o urbanos, donde tampoco fue un determinante del desempeño académico ${ }^{(21)}$. Esto puede deberse, en parte, a que el Perú se encuentra en un proceso de homogenización de las características universitarias, las cuales deben ser cumplidas independientemente de la región geográfica de procedencia.

La presente investigación brinda por primera vez evidencia de los determinantes que predisponen el poder adjudicar una plaza para obstetras en el SERUMS en obstetricia, el cual llega a ser el primer trabajo 
clínico de muchos profesionales. Asimismo, se deben considerar ciertas limitaciones como la posibilidad de que algunos egresados con bajas calificaciones no hayan postulado, debido a que presentan menores probabilidades de ingresar al SERUMS; habiendo así una posible subestimación de la proporción de profesionales que no son aptos.

\section{CONCLUSIÓN}

Concluimos que, mediante un modelo multivariado, el haber estudiado la carrera universitaria de obstetricia en una universidad pública y en la capital del país son factores que predisponen a tener mayor calificación académica en el examen nacional y en el promedio universitario, así como ser aptos para adjudicar una plaza en el SERUMS.
Contribuciones de autoría: Los autores participaron en la génesis de la idea, diseño de proyecto, recolección e interpretación de datos, análisis de resultados y preparación del manuscrito del presente trabajo de investigación.

\section{Financiamiento: Autofinanciado.}

Conflicto de interés: Los autores declaran no tener conflicto de interés en la publicación de este artículo.

Recibido: 17 de febrero 2020

Aprobado: 19 de marzo 2020

Correspondencia: Victor Hugo Moquillaza Alcántara.

Dirección: Av. Honorio Delgado 430, San Martín de Porres, Lima-Perú.

Teléfono: (+051) 982065404

Correo: victor.moquillaza@upch.pe

\section{REFERENCIAS BIBLIOGRÁFICAS}

1. El Peruano. Ley 30220: Ley universitaria. Superintendencia Nacional de Educación. Lima, Perú. 9 de Julio del 2014. Disponible en: https://www. sunedu.gob.pe/wp-content/uploads/2017/04/Ley-universitaria-30220.pdf

2. Razo-Abundis IY, Dibut-Toledo LS, Íñigo-Bajo ER. Aseguramiento de la calidad en la educación superior: experiencia de la universidad del golfo de California México. Revista Universidad y Sociedad. 2016; 8(1). Disponible en: http:// scielo.sld.cu/scielo.php?script=sci_arttext\&pid=S2218-36202016000100028

3. Superintendencia Nacional de Educación. Universidades: Públicas y privadas. [Página web] Lima, Perú. Citado el 22 de Noviembre del 2019. Disponible en: https://www.sunedu.gob.pe/lista-universidades/

4. El Peruano. Oficializan el Examen Nacional de Obstetricia. Resolución Ministerial № 422-2016-MINSA.2016. Lima, Perú. 16 Junio del 2016. Disponible en web: https://busquedas.elperuano.pe/normaslegales/oficializan-elexamen-nacional-de-obstetricia-enaobs-resolucion-ministerial-no-4222016minsa-1393796-1/

5. Universidad de San Martin de Porres. Asociación Peruana de Escuelas y Facultades de Obstetricia ASPEFOBST desarrolló Segundo Examen Naciona de Obstetras. [Página web] Citado el 22 de Noviembre 2019. Disponible en web: https://www.usmp.edu.pe/index.php?pag=novedades\&sec=nov19_22

6. Mejia CR, Valladares-Garrido MJ, Romero BM, Valladares-Garrido D, LinaresReyes E. Accidentes laborales asociados al desánimo de médicos SERUMS para laborar en el primer nivel de atención de Lima, Perú. Revista Mexicana del Instituto Mexicano del Seguro Social. 2017; 55(6). Disponible: https://www. medigraphic.com/cgi-bin/new/resumen.cgi?IDARTICULO=76440

7. Mejia C, Quiñones-Laveriano DM. SERUMS y la migración de médicos: propósito de una cohorte de médicos de Lima. Rev Per Med Exp Salud Pública. 2015; 32(2). Disponible en: http://www.scielo.org.pe/scielo.php?pid=S1726$46342015000200037 \&$ script=sci_arttext\&tlng=pt

8. Mejia C, Inga-Berrospi F, Albrecht-Lezama C, Quispe-García AA. Problemas laborales en plazas del Servicio Rural Urbano-Marginal en Salud (SERUMS) auto-reporte de los médicos. Acta Médica Peruana. 2016; 33(1). Disponible en: https://www.redalyc.org/pdf/966/96645712014.pdf

9. Mujica O, Pereira-Victorio CJ, Munayco C. Sobre el impacto redistributivo del SERUMS en la disponibilidad de médicos en Perú. Rev Per Med Exp Salud Pública. 2015; 32(1): 191-204. Disponible en: https://www.scielosp.org/pdf/ rpmesp/2015.v32n1/193-194/es

10. Mayta-Tristán P, Poterico J, Galán-Rodas E, Raa-Ortiz D. ¿Existe equidad injusta por la eliminación del requisito obligatorio del Serums? Rev Per Med Exp Salud Pública. 2015; 32(1): 191-204. Disponible en: https://www.scielosp. org/pdf/rpmesp/2015.v32n1/195-196/es
11. Taype-Rondán A, Alfaro-Toloza P. Falencias en el sistema de adjudicación de plazas del Servicio Rural Urbano Marginal en Salud en el Perú. Rev Med Hered. 2015; 26(2). Disponible en: http://www.scielo.org.pe/scielo.php?pid=S1018130X2015000200010\&script=sci_arttext

12. Ministerio de Salud. Serums: Proceso 2019. Dirección general de personal de salud. [Página web] Lima, Perú. Citado el 22 de Noviembre del 2019. Disponible en: https://www.minsa.gob.pe/dggdrh/comunicate/antes.html

13. Diario La República. ¿Particular o nacional? [Página web] Lima, Perú. Citado el 22 de Noviembre del 2016. Disponible en: https://larepublica.pe/ archivo/327743-particular-o-nacional/

14. Macknight AD. What determines the physiology that students learn? Advances in Physiology Education. 2019; 43(2): 172-174. DOI: https://doi. org/10.1152/advan.00035.2019

15. Rodrigues-Marques M, Monteiro LM, Dos Santos M, Pessoa B, Da Mota M dos Reis RS. Risk and prevalence of anemia among women attending public and private universities. Ecol Food Nutr. 2015; 54(5): 520-8. DOl: https://doi.org $/ 10.1080 / 03670244.2015 .1037442$

16. Alves G, Cardoso-Ferreira MB, Neves-Nunes L, Marques-Ribeiro MF Pedagogical training profile of basic health sciences faculty in biomedical and related fields at Brazilian public and private higher education institutions. Advances in Physiology Education. 2019; 43(29): 180-190. DOl: https://doi. org/10.1152/advan.00211.2018

17. Mejía-Navarrete J. El proceso de la educación superior en el Perú: La descolonialidad del saber universitario. Cinta de Moebio. 2018; 61(1): 56-71. DOI: http://dx.doi.org/10.4067/S0717-554X2018000100056

18. Espinosa O. Educación superior para indígenas de la Amazonía peruana: balance y desafíos. Anthropologica. 2017; 35(39). DOl: http://dx.doi. org/10.18800/anthropologica.201702.005

19. Parker PD, Jerrim J, Anders J, Astell-Burt T. Does Living Closer to a University Increase Educational Attainment? A Longitudinal Study of Aspirations, University Entry, and Elite University Enrolment of Australian Youth. J Youth Adolesc. 2016; 45(69): 1156-75. DOI: https://doi.org/10.1007/s10964-0150386- $x$

20. Hendrick CE, Cohen AK, Deardorff J, Duncan-Cance J. Biological and socio-cultural factors during the school years predicting women's lifetime educational attainment. J Sch Health. 2016; 86(3): 215-224. DOl: https://doi. org/10.1111/josh.12368

21. Carrasco-Cortez V, Lozano-Salazar E, Velásquez-Pancca E. Análisis actual y prospectivo de la oferta y demanda de médicos en el Perú 2005-2011. Acta Médica Peruana. 2008; 25(1). Disponible en: http://www.scielo.org.pe/scielo. php?script=sci_arttext\&pid=S1728-59172008000100005 